\title{
Noncompliance with Prescribed Eyedrop Regimens Among Patients Undergoing Cataract Surgery-Prevalence, Consequences, and Solutions
}

\author{
Cynthia Matossian \\ Temple University School of Medicine, Philadelphia, PA, USA
}

DOI: https://doi.org/10.17925/USOR.2020.13.1.18

F ollowing cataract surgery, patients are typically prescribed antibiotic, non-steroidal anti-inflammatory drug and corticosteroid eyedrops, each of which may be administered with a different frequency. However, patient noncompliance with prescribed eyedrop treatment is prevalent and may lead to a less than ideal outcome, with potential complications such as infection or inflammation. Numerous studies have been conducted to evaluate the extent of patient noncompliance and to identify contributing factors. The majority of these studies have focused on patients with glaucoma, because it is a chronic disease requiring lifelong use of eyedrops. Patients who have undergone cataract surgery may face greater challenges with drop instillation than glaucoma patients because of limited experience with eyedrops prior to surgery. Additionally, cataracts most often affect older persons, in whom physical or cognitive limitations may further impede successful eyedrop administration. New methods of drug delivery that bypass patient compliance are currently available. They include surgery with fewer drops as well as medication delivery systems injected or deposited into ocular structures perioperatively. These drug delivery methods not only reduce topical drop administration, but have supplanted eyedrop use in many countries. Alternative drug delivery systems are increasingly being adopted in the USA.

\section{Keywords}

Cataract, eyedrops, drop compliance, medication adherence, instillation, dropless surgery, noncompliance, non-adherence, drop regimen

Disclosure: Cynthia Matossian is a consultant for EyePoint Pharmaceuticals, the company that manufactures Dexycu ${ }^{\circledast}$, a US Food and Drug Administration-approved 9\% dexamethasone intraocular suspension indicated for the intracameral treatment of inflammation after eye surgery.

Acknowledgments: Assistance with manuscript preparation was provided by Ethis, Inc., which was supported by EyePoint Pharmaceuticals. The views and opinions expressed are those of the author and do not necessarily reflect those of EyePoint Pharmaceuticals.

Review Process: Double-blind peer review.

Compliance with Ethics: This study involves a review of the literature and did not involve any studies with human or animal subjects performed by the author.

Authorship: The named author meets the International Committee of Medical Journal Editors (ICMJE) criteria for authorship of this manuscript, takes responsibility for the integrity of the work as a whole, and has given final approval for the version to be published.

Access: This article is freely accessible at touchOPHTHALMOLOGY.com (C) Touch Medical Media 2020

Received: October 21, 2019

Accepted: February 26, 2020

Published Online: April 24, 2020

Citation: US Ophthalmic Review. 2020;13(1):18-22

Corresponding Author: Cynthia Matossian, Temple University School of Medicine, Philadelphia, PA, USA; Matossian Eye Associates, 501 Hyde Park, Route 202, Doylestown, PA 18902, USA.

E: cmatossian@matossianeye.com

Twitter: @MatossianEye

Support: No funding was received in the publication of this article.
Cataract is the leading cause of blindness worldwide and accounts for half of all visual impairment in the USA. ${ }^{1}$ Contributing risk factors are older age, smoking, alcohol use, sunlight exposure, and diabetes. ${ }^{1}$ Cataract affects more than 24 million Americans; that number is expected to reach 50 million by the year 2050. ${ }^{1}$ More than 3.6 million cataract surgeries are performed every year in the USA. The prescription of antibiotic eyedrops for the prevention of infection, and the prescription of steroid or non-steroidal anti-inflammatory drug (NSAID) eyedrops for the prevention of inflammation, are both well-accepted practice for the immediate pre- and postoperative period. The reasons for this are clear: eyedrops are a well-established, convenient, noninvasive method of delivering drugs to the anterior segment of the eye. ${ }^{2}$ In contrast, patient non-adherence with recommended medications may negatively impact clinical outcomes. ${ }^{3}$ The average noncompliance rate for eyedrop treatment has been shown to be approximately $30 \% .{ }^{4}$ Challenges posed by eyedrop noncompliance and the benefits of new drug delivery systems for patients undergoing cataract surgery will be discussed in this narrative review. Our goal is to evaluate the traditional role of topical prophylaxis in cataract surgery and compare it to the new era of intraocular (intracameral and intravitreal) or intracanalicular drug delivery options.

\section{Literature search}

PubMed and Google Scholar were searched for English-language articles on the problem of noncompliance with eyedrops, using the search terms "eyedrop (or eye drop)," "compliance," "adherence," "cataract," "post-cataract prophylaxis," "dropless surgery," and "intracameral injection." We selected search terms that would be broad enough to encompass the various aspects of our topic yet narrow enough to focus on our specific objectives for the study - to evaluate the role of perioperative prophylaxis 
in cataract surgery. No publication date filters were applied; however, the focus was on articles published since 2000. Some earlier, seminal articles (e.g., Apt et al., ${ }^{5}$ Winfield et al., ${ }^{6}$ Burns et al. ${ }^{7}$ ) were also included. In addition, reference lists of the most relevant papers were reviewed. Articles that described mechanical devices and the use of illustrations or educational techniques to improve compliance were excluded, as they were considered to be outside the scope of this paper.

\section{Eyedrops-the challenges}

The intrinsic characteristics of eyedrops and problems of absorption across the cornea and conjunctiva to reach target tissues, pose some of the biggest challenges. Only $1-7 \%$ of the instilled drug makes it through the cornea to reach the aqueous humor due to the hurdles posed by the precorneal tear clearance mechanism, the corneal-epithelial barrier, and drug loss through the conjunctival-scleral route. ${ }^{2}$ The $\mathrm{pH}$ affects drug ionization and interferes with drug absorption: a pH outside of the normal range increases reflex tearing and blinking, contributing to dilution and loss of drug via nasolacrimal drainage..$^{2,8}$

To help with the recognized absorption challenges, hydrophobic alcohol-based and acetate suspensions were designed. These penetrate all layers of the cornea more efficiently than hydrophilic formulations. However, they require shaking to reach homogeneity. Asking patients to shake the product long enough to achieve the desired consistency has not shown to be effective. ${ }^{5}$ Failure to shake suspensions leads to variability in active drug delivery to target tissues. Another issue is ocular surface toxicity from preservatives. Multidose containers often require a preservative to prevent microorganism growth within the bottle; the most commonly used preservative, benzalkonium chloride, enhances corneal permeability of various drugs but may be toxic to the corneal and conjunctival epithelium. ${ }^{2}$

\section{Compliance}

Compliance is a complex healthcare concept comprising multiple patient factors. These may include illness and health beliefs, degree of self-efficacy, communication styles, cognitive factors, social support, trust in healthcare providers, literacy, race, income, and age. ${ }^{9}$ The definition of noncompliance is not standardized; different studies have used different definitions of the term. Generally, it is understood to be a failure to conform to recommendations made by the provider with respect to timing, dosage, and frequency of medication use.,.9 The average noncompliance rate for eyedrop treatment has been shown to be approximately $30 \%{ }^{4}$ Poor compliance can impact clinical outcomes. ${ }^{3}$

Behavioral scientists have described two categories of noncompliance: unintentional and intentional. ${ }^{10}$ Whereas intentionally noncompliant patients may decide for a variety of reasons to disregard or amend recommendations for treatment, unintentional noncompliance is passive and may manifest as forgetfulness, misunderstanding, or lack of awareness. ${ }^{10}$ With respect to eyedrops, noncompliance is most often unintentional and includes diminished ability or inability to properly instill the medication into the eye.

\section{Instillation technique}

Appropriate eyedrop instillation requires users to: (1) fill the prescription; (2) instill the correct number of drops, usually a single drop; (3) instill the drop(s) at the correct time(s) during the day; (4) shake the bottle if instructed to do so; and (5) repeat the dosing each day. ${ }^{11-13}$ Instillation technique is complex, must be taught, takes practice, and is neither intuitive nor easy to master. It requires manual dexterity, hand-eye coordination, and good vision. ${ }^{14}$ Blurring of vision and burning from the medication itself can trigger tearing and interfere with drop instillation.

In studies of patients with glaucoma, numerous attempts were required before drops successfully reached their target. Often, patients were unable to control the number of drops entering the eye. Instilling too many drops, or a stream of drops, may cause systemic adverse effects, particularly if instillation is not followed by punctal occlusion..$^{13}$ Overdosing represents wastage and additional costs. It also generates phone calls to the eyecare provider's office for refills and, frequently, denials by insurance plans for additional bottles within the specified timelines. ${ }^{15}$ In addition, spacing between eyedrops is key for ideal absorption. Finally, it is important to instill drops into the eye without touching the eye or adnexa with the bottle tip. Contamination of the tip can lead to an increased risk of infection. Corneal abrasions from a bottle tip have also been reported. ${ }^{13,16}$ Devices to assist patients with drop administration have generally received mixed reviews and are underused. ${ }^{0.16}$

\section{Compliance in patients undergoing cataract surgery}

Numerous studies have described the problems experienced by patients during eyedrop administration, ${ }^{11,13,17}$ and have identified and categorized the risk factors leading to noncompliance..$^{718-21}$ The majority of these studies have been conducted in patients with glaucoma.

Complex regimens cause confusion and lead to noncompliance. It is not uncommon for patients undergoing cataract surgery to be prescribed antibiotic, steroid, and NSAID drops postoperatively, resulting in a total of three separate bottles. Often, each of these medications has a unique dosing schedule requiring administration multiple times per day. This may be further complicated by a separate schedule following second-eye cataract surgery. Steroid drop schedules are the most challenging for patients since, unlike antibiotic drops, the dosing is tapered over time. A further complication in eyedrop delivery is the instruction to wait $\geq 5$ minutes before instilling different drops into the same eye. Patients undergoing cataract surgery who are on concomitant eyedrops for glaucoma, dry eye disease, or other ophthalmic conditions may be managing up to 14 drops per day from four or more different bottles, each medication with its own dosing schedule. Pharmacists may further confuse the patient by making generic substitutions that alter the dosing schedule from the surgeon's original prescription. Many physicians provide pictures of the recommended bottles, along with the dosing schedule as instructional aids for their patients. However, due to medication substitutions, patients may have difficulty following the instructions despite the provided visual aids. ${ }^{3}$

Unlike patients with glaucoma, who take their eyedrops chronically, patients undergoing cataract surgery are likely to have little or no experience with eyedrop use. ${ }^{22}$ In a prospective observational study conducted in three Canadian clinics 1 day after cataract surgery, 54 patients were asked to demonstrate their eyedrop instillation technique while being video recorded. Technique was evaluated on a binary scale, with points given for hand washing, location of drop application (eye, eyelid, cheek), total number of drops instilled, and bottle-tip contamination. Overall, 50 patients (92.6\%) missed their eye, 
instilled an incorrect number of drops, contaminated the bottle tip, or failed to wash their hands before instillation. Of the 54 patients who participated in the study, 12 (22.2\%) washed their hands, 37 (68.5\%) got the drop into the eye, 37 (68.5\%) instilled the correct number of drops, and 23 (42.6\%) managed not to touch the bottle tip to the ocular adnexa. There was a large discrepancy between the patients' perceptions of their ability to perform correctly and the observed technique of eyedrop administration.22

In another post-cataract surgery study, conducted in Ghana, on postoperative day $1,48.17 \%$ of subjects touched the tip of the bottle to the eye or ocular adnexa, and $56.9 \%$ of subjects were unable to instill only a single drop of the medication. ${ }^{23}$ In this study, as in other studies, patients believed they were proficient in instilling their eyedrops; however, their perception did not agree with those of the objective observers. ${ }^{23}$

\section{Compliance and increased age}

Age is the biggest risk factor associated with cataracts. By age $80,70 \%$ of white Americans, $61 \%$ of Hispanic Americans, and $53 \%$ of black Americans have cataracts. ${ }^{1}$ Unfortunately, older patients are the most likely to have difficulties with eyedrop administration, including problems with manual dexterity, lack of tactile sensibility, tremor of the hands or head, difficulty in tilting the head back, or various degrees of visual impairement. ${ }^{24}$ Correct opening and successful squeezing of eyedrop containers may present a manual problem for older patients, even when they are free of other physical conditions hindering self-application. ${ }^{24,25}$ Rheumatoid arthritis, osteoarthritis, carpal tunnel syndrome, and stroke are all associated with decreased finger strength. Neurologic diseases can cause deficits in control and direction of force application, which are required to coordinate functional tasks such as squeezing a bottle. ${ }^{6,25}$

Other limiting factors in the instillation of an eyedrop are the ability to lift a hand to the face and maintain aim, coordination, and fine motor control as the bottle is directed close to the eye. For many older patients, performing these tasks while maintaining a pinched grip may be next to impossible. Some elderly or disabled patients are unable to instill eyedrops on their own and require a caregiver to assist them, which exacerbates the perceived burden of eyedrop therapy while heightening stress during the perioperative period.? Other factors to consider among this age group are impaired visual acuity and concomitant diseases that limit sight. Therefore, these patients may encounter difficulty identifying each of the prescription eyedrop bottles and may mix them up due their inability to read the small print on the bottle labels. ${ }^{25}$

In addition to physical limitations, elderly patients may have cognitive or memory problems that make it difficult for them to follow a complex eyedrop regimen. ${ }^{3}$ Many older patients live alone and have no choice but to self-administer their eyedrops. It is highly unlikely that they are accurately complying with medical recommendations, even when they believe that they are doing so. ${ }^{7}$ These patients may already be managing multiple doses of systemic medications and may find themselves overwhelmed and unable to integrate their postoperative eyedrops into an existing schedule.

\section{Financial burden}

One of the key reasons for patient noncompliance with eyedrops is the high cost of the medications. For example, a typical pre- and post-surgery protocol is likely to include a quinolone antibiotic, an NSAID, and a corticosteroid, to be instilled up to four times daily for up to 4 weeks post-surgery. The average retail prices of eyedrops are as follows: approximately $\$ 191$ for $3 \mathrm{~mL}$ of a branded antibiotic eyedrop; approximately $\$ 288$ for $3 \mathrm{~mL}$ of a branded NSAID; and approximately $\$ 209$ for $5 \mathrm{~g}$ of a branded corticosteroid. ${ }^{26-28}$ Generics may be substituted at a lower cost; their prices, however, are increasing rapidly. ${ }^{29}$

Eyedrop prices vary depending on the location and the pharmacy. Coupons are often available. The out-of-pocket cost to the consumer may depend on age (Medicare eligible), income (Medicaid eligible), and type of commercial insurance. Surveys show that the retail cost of the drug combination used for post-cataract therapy ranges from a low of $\$ 175$ to a high of $\$ 431$ per eye, with a weighted average of $\$ 323$. Much of this cost is borne by Medicare or Medicaid. ${ }^{30}$ Nevertheless, for some, out-of-pocket co-payments can be as much as $\$ 650$ per eye. ${ }^{30}$ It is clear that the standard post-surgery protocol represents a considerable financial burden to many patients.

Furthermore, drops are often wasted in failed attempts at instillation. In such cases, an additional prescription may be required. When patients run out of their prescriptions early, most insurance companies deny approval for additional bottles, adding to the out-of-pocket expenses. ${ }^{15,30}$ Reports of patients delaying second-eye surgery due to concerns about drug costs are, therefore, not surprising.

\section{Consequences of poor compliance with eyedrops}

Antibiotic eyedrops are prescribed off label to prevent endophthalmitis, a devastating though rare infectious complication of surgery. A recent analysis of medical records revealed 112 endophthalmitis cases among 480,104 cataract procedures, indicating a postoperative endophthalmitis incidence of $0.023 \%$. Prognosis is generally uncertain and visual outcomes can be poor. ${ }^{31,32}$

Steroids and/or NSAIDS are prescribed to minimize inflammation and the development of cystoid macular edema (CME). ${ }^{33}$ During cataract surgery, trauma to the ocular tissues can trigger inflammation, pain, and in some cases, CME. Although CME usually resolves over time, it can permanently impact contrast sensitivity and visual quality. Even low to moderate levels of inflammation may temporarily reduce vision in the early postoperative period. The prevalence of post-cataract clinical CME with decreased visual acuity is between $1 \%$ and $2 \% .^{33}$

\section{Solutions to noncompliance with eyedrops}

Although surgical techniques have become considerably refined over the last few decades, it is only in recent years that the same attention has been placed on postoperative drop minimization to enhance the surgical journey. Approaches to post-surgical eyedrop reduction include topical compounded combination drops, compounded injectable medications, and reservoir-based drug delivery systems. Injectable medications and reservoir-based drug delivery systems may differ from one another by the technique and location of the drug or drug deposit.

Triamcinolone acetonide, moxifloxacin hydrochloride, with or without vancomycin, a compounded combination of antibiotic and steroid, became available in 2014 as a "dropless" approach. The combination is typically delivered to the vitreous by transzonular administration or 
through a pars plana approach. Studies have shown this strategy to be safe and efficacious in minimizing inflammation and infection. ${ }^{34}$ However, there are concerns with compounded products, include the possibility of mixing errors, contamination, and lack of US Food and Drug Administration (FDA) oversight.

Another option is a simplified "less-drops" postoperative regimen of compounded eyedrop therapy. In addition to improving compliance, reducing the number of drops may improve ocular surface toxicity by decreasing exposure to preservatives. This strategy may also reduce drug dilution by eliminating consecutive drop instillations. ${ }^{34}$ However, the same concerns over compounded injectable medications apply to compounded combination drops.

In contrast to compounded injectables or combination drops are the new FDA-approved reservoir-based drug delivery systems. These dexamethasone preparations are used at the conclusion of eye surgery to eliminate the need for postoperative steroid drops. One steroid preparation is designed for intracameral placement of a dexamethasone spherule in the sulcus under the iris; the second steroid delivery is a small rod-like implant designed for placement into the inferior canaliculus. Both systems act as a drug depot to deliver higher levels of dexamethasone initially with a gradual taper over 30 days. ${ }^{35-37}$

Dexycu $^{\circledast} \quad$ (dexamethasone intraocular suspension 9\%; EyePoint Pharmaceuticals, Watertown, MA, USA) delivers a biodegradable extended-release formulation of dexamethasone into the posterior chamber via a single injection at the end of surgery. Dexycu was evaluated in a randomized, double-masked, placebo-controlled trial that assessed the proportion of patients ( $n=394)$ with anterior chamber cell clearing (i.e., cell score=0) on postoperative day 8 (primary endpoint). Compared with placebo, a greater percentage of patients treated with Dexycu had anterior chamber clearing at day 8 (about 60\% in the Dexycu $517 \mu \mathrm{g}$ group versus $20 \%$ in the placebo group). Fewer inflammatory adverse effects were reported in the Dexycu group compared with the placebo group, and no serious ocular adverse events were reported up to postoperative day $90 .{ }^{35}$ In a second study, the safety and efficacy of Dexycu and prednisolone acetate $1 \%$ were found to be similar, although patients expressed a strong preference for Dexycu based on its greater convenience. ${ }^{36}$

Dextenza ${ }^{\circledast}$ (dexamethasone ophthalmic insert 0.4 mg; Ocular Therapeutix Bedford, MA, USA) is inserted into the lower canaliculus of the eyelid. The hydrogel implant releases dexamethasone towards the surface of the eye once activated by the tear film. The approval was based on data from two phase III trials, in which it was associated with a higher incidence of pain-free patients at all postoperative days (days $2,4,8$ ) compared with vehicle. At day 8, in studies 1 and 2, respectively, $80 \%$ and $77 \%$ of Dextenza-treated patients were pain free versus $43 \%$ and $59 \%$ for vehicle. Transient intraocular pressure (IOP) increases of $\geq 10 \mathrm{mmHg}$ in the study eye were seen in a similar proportion of patients in the Dextenza groups $(6.8 \%$ in study 1 and $4.4 \%$ in study 2) compared with the placebo groups (3.6\% and $5.0 \%$, respectively). ${ }^{37}$

Potential IOP elevation by a depot steroid delivery system has been raised as a concern by some surgeons. It is therefore recommended to avoid these products in patients who are steroid responders or who have a history of glaucoma or ocular hypertension. ${ }^{37}$ The Dextenza insert can be irrigated out of the eye if necessary. No long-term steroid response issues were noted with either drug delivery system in their respective clinical trials. In addition, the use of intraocular antibiotics with cataract surgery is increasing. In Europe, topical antibiotic prophylaxis has nearly disappeared; intraocular delivery has become universal practice in Sweden, is considered standard of care in France and Denmark, and is recommended by national societies in several countries. ${ }^{38}$ In Australia, intraocular antibiotic use exceeds $80 \% .{ }^{39}$ In the USA, this practice has not yet become routine due to the lack of an FDA-approved antibiotic formulation for intraocular use. Regardless, the use of intracameral antibiotics in the USA is on the rise.

\section{Financial ramifications of the FDA-approved alternative steroid delivery models}

One of the motivators for the development of alternative steroid delivery systems was to reduce the financial burden borne by patients. Both of these novel steroid depot products, Dexycu and Dextenza, were awarded pass-through status. ${ }^{40}$ Transitional pass-through payments-Medicare reimbursement paid on top of an ambulatory surgery center's fee for a limited amount of time-were established by congress to foster innovative medical devices, drugs and biologicals. The facility or institution purchases the medication and is then reimbursed by Medicare. Patients have reported satisfaction with both the increased convenience and reduced financial burden. ${ }^{34,36}$

\section{Conclusion}

Eyecare professionals have long been cognizant of the serious ramifications of noncompliance with prescription eyedrops while readily admitting to being unaware of its true extent. Over the last decade, great strides have been made to simplify eyedrop regimens, but the physical and cognitive limitations, especially common in older patients remain an obstacle to optimal therapy. With the recent availability of two FDA-approved steroid delivery systems for patients undergoing ocular surgery, the burden of drops can be reduced while enhancing their postoperative journey. $\square$
1. Olson RJ, Braga-Mele R, Chen SH, et al. Cataract in the adult eye preferred practice pattern. Ophthalmology. 2017;124:P1-119.

2. Ghate D, Edelhauser HF. Ocular drug delivery. Expert Opin Drug Deliv. 2006;3:275-87.

3. Vermeire $\mathrm{E}$, Hearnshaw $\mathrm{H}$, Van Royen $\mathrm{P}$, et al. Patient adherence to treatment: three decades of research. A comprehensive review. J Clin Pharm Ther. 2001;26:331-42.

4. Vandenbroeck S, De Geest S, Dobbels S, et al. Prevalence and correlates of self-reported nonadherence with eye drop treatment: The Belgian Compliance Study in Ophthalmology J Glaucoma. 2011:20:414-21.

5. Apt L, Henrick A, Silverman LM. Patient compliance with use of topical ophthalmic suspensions. Am J Ophthalmol. 1979;87:210-4.

6. Winfield AJ, Jessiman D, Williams A, et al. A study of the causes of non-compliance in patients prescribed eyedrops. Br J Ophthalmol. 1990;74:477-80.

7. Burns E, Mulley GP. Practical problems with eye-drops among elderly ophthalmology outpatients. Age Ageing. 1992;21:168-70.

8. Coffey MJ, DeCory HH, Lane SS. Development of a non-settling ge formulation of $0.5 \%$ loteprednol etabonate for anti-inflammatory use as an ophthalmic drop. Clin Ophthalmol. 2013;7:299-312.

9. Dreer LE, Girkin C, Mansberger SL. Determinants of medication adherence to topical glaucoma therapy. Glaucoma. 2012;21:234-40.

10. Rees $\mathrm{G}$, Leong $\mathrm{O}$, Crowston JG, et al. Intentional and nonintentional nonadherence to ocular hypotensive treatment in patients with glaucoma. Ophthalmology. 2010;117:903-8.

11. Tsai T, Robin AL, Smith III JP. An evaluation of how glaucoma patients use topical medications: a pilot study. Trans Am Ophthalmol Soc. 2007:105:29-35.

12. Muir KW, Lee PP. Glaucoma medication adherence: room for improvement in both performance and measurement. Arch Ophthalmol. 2011;129:243-5.

13. Stone JL, Robin AL, Novack GD, et al. An objective evaluation of eyedrop instillation in patients with glaucoma. Arch Ophthalmol. 2009;127:732-6.

14. Robin A, Grover DS. Compliance and adherence in glaucoma management. Indian J Ophthalmol. 2011;59(Suppl.1):S93-6.

15. Eaton AM, Gordon GM, Konowal A, et al. A novel eye drop application monitor to assess patient compliance with a prescribed regimen: a pilot study. Eye. 2015;29:1383-91

16. Davies I, Williams AM, Muir KW. Aids for eye drop administration. Surv Ophthalmol. 2017;62:332-45.

17. Gao X, Yang Q, Huang W, et al. Evaluating eye drop instillation technique and its determinants in glaucoma patients. J Ophthalmol. 2018:1376020.

18. Friedman DS, Okeke $C O$, Jampel $H D$, et al. Risk factors for poor adherence to eyedrops in electronically monitored patients with glaucoma. Ophthalmology. 2009;116:1097-105.

19. Kholdebarin R, Campbell RJ, Jin Y-P, et al. for the Canadian Compliance Study Group. Multicenter study of compliance 
and drop administration in glaucoma. Can J Ophthalmol. 2008;43:454-61.

20. Newman-Casey PA, Robin AL, Blachley T, et al. Most common barriers to glaucoma medication adherence: a cross-sectional survey. Ophthalmology. 2015;122:1308-16.

21. Tsai JC, MCClure CA, Ramos SE, et al. Compliance barriers in glaucoma: a systematic classification. J Glaucoma. 2003;12:393-8.

22. An JA, Kasner O, Samek DA, et al. Evaluation of eyedrop administration by inexperienced patients after cataract surgery. J Cataract Refract Surg. 2014;40:1857-61

23. Liu Y, Murdoch A, Bassett K, et al. Proficiency of eyedrop instillation in postoperative cataract patients in Ghana. Clin Ophthalmol. 2013;7:2099-105.

24. Dietlein TS, Jordan JF, Lüke C, et al. Self-application of single-use eyedrop containers in an elderly population: comparisons with standard eyedrop bottle and younger patients. Acta Ophthalmol. 2008;86:856-9.

25. Connor AJ, Severn PS. Force requirements in topical medicine use - the squeezability factor. Eye. 2011;25:466-9.

26. Drugs.com. Vigamox Prices, Coupons and Patient Assistance Programs. Available at: www.drugs.com/price-guide/vigamox (accessed March 5, 2020).

27. Drugs.com. Prolensa Prices, Coupons and Patient Assistance Programs. Available at: www.drugs.com/price-guide/prolensa (accessed March 5, 2020)

28. Drugs.com. Lotemax Prices, Coupons and Patient Assistance Programs. Available at: www.drugs.com/price-guide/lotemax (accessed March 5, 2020).

29. Loden JC. Dropless cataract surgery. Better for the patient, bette for the surgeon. Ophthalmol Mgmt. 2014;18:20-2.

30. Analysis of the Economic Impacts of Dropless Cataract Therapy on Medicare, Medicaid, State Governments, and Patient Costs. Andrew Chang \& Co, LLC. Sacramento, CA. 2015. Available at: https://stateofreform.com/wp-content/ uploads/2015/11/CSIE_Dropless_Economic_Study.pdf (accessed September 6, 2019).

31. Jabbarvand $\mathrm{M}$, Hashemian $\mathrm{H}$, Khodaparast $\mathrm{M}$, et al. Endophthalmitis occurring after cataract surgery: outcomes of more than 480000 cataract surgeries, epidemiologic features, and risk factors. Ophthalmology. 2016;123:295-30.

32. Kernt M, Kampik A. Endophthalmitis: pathogenesis, clinical presentation, management, and perspectives. Clin Ophthalmol. 2010;4:121-35.

33. Henderson BA, Kim JY, Ament CS, et al. Clinical pseudophakic cystoid macular edema. Risk factors for development and duration after treatment. J Cataract Refract Surg. 2007;33:1550-8.

34. Fisher BL, Potvin R. Transzonular vitreous injection vs. a single drop compounded topical pharmaceutical regimen after cataract surgery. Clin Ophthalmol. 2016;10:1297-303.

35. Donnenfeld $\mathrm{E}$, Holland E. Dexamethasone intracameral drug-delivery suspension for inflammation associated with cataract surgery: a randomized, placebo-controlled, phase III trial. Ophthalmology. 2018;125:799-806.

36. Donnenfeld ED, Solomon KD, Matossian C. Safety of IBI-10090 for inflammation associated with cataract surgery: phase 3 multicenter study. J Cataract Refract Surg. 2018;44:1236-46.

37. Walters T, Bafna S, Vold S, et al. Efficacy and safety of sustained release dexamethasone for the treatment of ocular pain and inflammation after cataract surgery: results from two phase 3 studies. I Clin Exp Ophthalmol 2016:7: DOl: 10.4172/2155-9570.1000572.

38. Javitt JC. Intracameral antibiotics reduce the risk of endophthalmitis after cataract surgery. Does the preponderance of the evidence mandate a global change in practice? Ophthalmology. 2016;123:226-30.

39. George NK, Stewart MW. The routine use of intracameral antibiotics to prevent endophthalmitis after cataract surgery: how good is the evidence? Ophthalmol Ther. 2018;7:233-45.

40. Wiley WF. Adversity drives cataract surgery innovation. ophthalmology Mgmt. 2019;23:36-41. 